\title{
Article \\ Third Hankel Determinant for a Subclass of Univalent Functions Associated with Lemniscate of Bernoulli
}

\author{
Najeeb Ullah ${ }^{1}$, Irfan Ali ${ }^{1}$, Sardar Muhammad Hussain ${ }^{1}$, Jong-Suk Ro ${ }^{2,3}, * \mathbb{D}$, , Nazar Khan ${ }^{4}$ (D) and Bilal Khan ${ }^{5}(\mathbb{D}$ \\ 1 Department of Mathematical Sciences, Balochistan University of Information Technology, \\ Engineering and Management Sciences (BUITEMS), Quetta 87300, Pakistan; \\ Najeebullah.Kakar@buitms.edu.pk (N.U.); irfan.ali@buitms.edu.pk (I.A.); smhussain01@gmail.com (S.M.H.) \\ 2 School of Electrical and Electronics Engineering, Chung-Ang University, Dongjak-gu, Seoul 06974, Korea \\ 3 Department of Intelligent Energy and Industry, Chung-Ang University, Dongjak-gu, Seoul 06974, Korea \\ 4 Department of Mathematics, Abbottabad University of Science and Technology, Abbottabad 22010, Pakistan; \\ nazarmaths@gmail.com \\ 5 School of Mathematical Sciences and Shanghai Key Laboratory of PMMP, East China Normal University, \\ 500 Dongchuan Road, Shanghai 200241, China; bilalmaths789@gmail.com \\ * Correspondence: jongsukro@gmail.com
}

check for updates

Citation: Ullah, N.; Ali, I.; Hussain,

S.M.; Ro, J.-S.; Khan, N.; Khan, B.

Third Hankel Determinant for a Subclass of Univalent Functions Associated with Lemniscate of Bernoulli. Fractal Fract. 2022, 6, 48. https://doi.org/10.3390/

fractalfract6010048

Academic Editor: Acu Mugur Alexandru; Shahram Najafzadeh

Received: 28 December 2021

Accepted: 14 January 2022

Published: 16 January 2022

Publisher's Note: MDPI stays neutral with regard to jurisdictional claims in published maps and institutional affiliations.

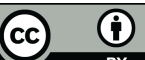

Copyright: (c) 2022 by the authors Licensee MDPI, Basel, Switzerland. This article is an open access article distributed under the terms and conditions of the Creative Commons Attribution (CC BY) license (https:// creativecommons.org/licenses/by/ $4.0 /)$.

\begin{abstract}
This paper deals with a new subclass of univalent function associated with the right half of the lemniscate of Bernoulli. We find the upper bound of the Hankel determinant $H_{3}(1)$ for this subclass by applying the Carlson-Shaffer operator to it. The present work also deals with certain properties of this newly defined subclass, such as the upper bound of the Hankel determinant of order 3, coefficient estimates, etc.
\end{abstract}

Keywords: Hankel determinant; Carlson-Shaffer operator; analytic functions; lemniscate of Bernoulli; starlike functions

\section{Introduction}

Suppose that $\mathcal{H}(E)$ represents the class of those functions that are analytic in any open unit disk, i.e.,

$$
E=\{z: z \in \mathbb{C} \text { such that }|z|<1\} .
$$

Here, $\mathbb{C}$ denotes the set of complex numbers.

In a similar way, we denote the class $\mathcal{A}$ of those analytic functions, which satisfies

$$
f(z)=z+\sum_{n=2}^{\infty} a_{n} z^{n} \quad(\text { for all } z \in E) .
$$

The class $\mathcal{A}$ is normalized by

$$
f(0)=0=f^{\prime}(0)-1 .
$$

Let us consider the analytic functions with the form

$$
p(z)=1+\sum_{n=1}^{\infty} p_{n} z^{n},
$$

are denoted by the class $\mathcal{P}$, such that

$$
\Re(p(z))>0 \quad \text { (for all } z \in E) .
$$

Moreover, here $\mathcal{S}$ represents the class of univalent function in $E$. We represent by $\mathcal{S}^{*}$, the class of starlike function in $E$, which satisfies 


$$
\frac{z f^{\prime}(z)}{f(z)} \in \mathcal{P} \quad(\text { for all } z \in E)
$$

Furthermore, $\mathcal{S} \mathcal{L}^{*}$ represents the class of those functions that satisfying

$$
\left.\left|\left(\frac{z f^{\prime}(z)}{f(z)}\right)^{2}-1\right|<1 \quad \text { (for all } z \in E\right) .
$$

Hence, $f \in \mathcal{S} \mathcal{L}^{*}$, iff, $\frac{z f^{\prime}(z)}{f(z)}$ is the inside region that is bounded by the right half of the lemniscate of Bernoulli, it can be expressed by

$$
\left|\omega^{2}-1\right|<1
$$

Sokól [1], and Sokól and Stankiewicz (see [2]) have introduced this class. One may represent subordination between any two analytic functions; $f$ and $g$ in $E$ as

$$
f(z) \prec g(z) \text { or } \quad f \prec g \text {. }
$$

If we have a Schwarz function $w$ in $E$, which is analytic and satisfying the following conditions

$$
|w(z)|<1 \quad \& \quad w(0)=0,
$$

implies

$$
f(z)=g(w(z))
$$

Furthermore, if $g$ satisfies the condition of univalent function in $E$, then the equivalence becomes

$$
f(z) \prec g(z) \quad(z \in E) \Rightarrow f(0)=g(0) \quad \& \quad f(E) \subset g(E) .
$$

Definition 1. Suppose that $S L^{*}(\alpha, \beta)$ is the subclass of analytic functions given by

$$
S L^{*}(\alpha, \beta)=\left\{f \in A:\left|\left(\frac{z[L(\alpha, \beta) f(z)]^{\prime}}{L(\alpha, \beta) f(z)}\right)^{2}-1\right|<1\right\},
$$

or

$$
\frac{z[L(\alpha, \beta) f(z)]^{\prime}}{L(\alpha, \beta) f(z)} \prec \sqrt{1+z} \quad(z \in E),
$$

where

$$
L(\alpha, \beta) f(z)=z+\sum_{n=2}^{\infty} \frac{(\alpha)_{n-1}}{(\beta)_{n-1}} a_{n} z^{n}
$$

and

$$
(x)_{n}=x(x+1)(x+2) \cdots(x+n-1)
$$

with

$$
(\alpha)_{1}=\alpha, \quad(\beta)_{1}=\beta,
$$

where

$$
(\alpha)_{2}=\alpha(\alpha+1), \quad(\beta)_{2}=\beta(\beta+1) .
$$


Suppose that $q \geq 1$ and $n \geq 0$. The definition of $q$ th Hankel determinant is given by

$$
H_{q}(n)=\left|\begin{array}{lllll}
a_{n} & a_{n+1} & \cdot & \cdot & a_{n+q-1} \\
a_{n+1} & \cdot & & & \cdot \\
\cdot & \cdot & & & \cdot \\
\cdot & \cdot & & & \cdot \\
\cdot & \cdot & & & \cdot \\
a_{n+q-1} & \cdot & \cdot & \cdot & a_{n+2(q-1)}
\end{array}\right| .
$$

Several authors worked on this determinant. Different authors [3-8] worked on $H_{2}$ (2) for various classes of functions and find its sharp upper bound. The functional $\left|a_{3}-a_{2}^{2}\right|=H_{2}(1)$ is known as a Fekete-Szegö functional. For any real and complex values of $\mu$, this functional was generalized as $\left|a_{3}-\mu a_{2}^{2}\right|$. For a class of univalent functions $f \in \mathcal{S}$ and some real values of $\mu$, the sharp estimates of $\left|a_{3}-\mu a_{2}^{2}\right|$ were evaluated by Fekete and Szegö, which is also known as functional $\left|a_{2} a_{4}-a_{3}^{2}\right|$ equivalent to $H_{2}(2)$. Similarly, for a subclass of analytic functions, the Hankel determinant of $H_{3}(1)$ was studied by Babalola [9]. Several authors (Refs. [10-12]) also studied the Hankel determinant $H_{3}(1)$. Our main focus in this work is for the class $S L^{*}(\alpha, \beta)$ on the Hankel determinant $H_{3}(1)$.

\section{Set of Lemmas}

Lemma 1 ([13]). Assuming that $p \in \mathcal{P}$ be the form of Equation (2), we may write

$$
\left|p_{2}-v p_{1}^{2}\right| \leq\left\{\begin{array}{lc}
-2+4 v, & v>1 \\
2, & 0 \leq v \leq 1 \\
2-4 v, & v<0
\end{array}\right.
$$

For $0<v<1$, the sharpness of the upper bound stated above may be enhanced by

$$
(1-v)\left|p_{1}\right|^{2}+\left|p_{2}-v p_{1}^{2}\right| \leq 2 \quad\left(\frac{1}{2}<v \leq 1\right)
$$

$\&$

$$
v\left|p_{1}\right|^{2}+\left|p_{2}-v p_{1}^{2}\right| \leq 2 \quad\left(0<v \leq \frac{1}{2}\right)
$$

Lemma 2 ([13]). Let us assume that $p \in \mathcal{P}$ be the form Equation (2), and for any complex number $v$, we have

$$
\left|p_{2}-v p_{1}^{2}\right| \leq 2 \max (1,|1-2 v|)
$$

Sharp results can be obtained by following

$$
p(z)=\frac{1+z}{1-z}
$$

and

$$
p(z)=\frac{1+z^{2}}{1-z^{2}}
$$

Lemma 3 ([14]). Let us assume that $p \in \mathcal{P}$ be the form Equation (2); then, we have

$$
p_{2}=4 x+(1-x) p_{1}^{2}
$$

for any $x$, such that $|x| \leq 1$

$$
p_{3}=\frac{p_{1}^{3}}{4}+\left[\frac{p_{1}}{2} x-\frac{p_{1}}{4} x^{2}+\frac{1}{2}\left(1-|x|^{2}\right) z\right]\left(4-p_{1}^{2}\right)
$$

for any $z$, if $|z| \leq 1$. 


\section{Main Results}

This section will provide proofs of the main results.

Theorem 1. Assuming that $L(\alpha, \beta) f(z) \in S L^{*}(\alpha, \beta)$ and is of the form (5). Then

$$
\left|a_{3}-\mu a_{2}^{2}\right| \leq \begin{cases}\frac{1}{16}\left(\frac{\beta(\beta+1)}{\alpha(\alpha+1)}-4 \mu \frac{\beta^{2}}{\alpha^{2}}\right), & \mu<-\frac{3}{4} \\ \frac{1}{4}, & -\frac{3}{4} \leq \mu \leq \frac{5}{4} \\ \frac{1}{16}\left(4 \mu \frac{\beta^{2}}{\alpha^{2}}-\frac{\beta(\beta+1)}{\alpha(\alpha+1)}\right), & \mu>\frac{5}{4}\end{cases}
$$

Proof. If $L(\alpha, \beta) f(z) \in S L^{*}(\alpha, \beta)$, then it follows from Equation (4) that

$$
\frac{z[L(\alpha, \beta) f(z)]^{\prime}}{L(\alpha, \beta) f(z)} \prec \Phi(z) .
$$

Let us define the function,

$$
p(z)=1+\sum p_{n} z^{n}=\frac{1+w(z)}{1-w(z)} .
$$

As $p \in \mathcal{P}$, so

$$
\frac{p(z)-1}{p(z)+1}=w(z)
$$

Using Equation (4), we have

$$
\frac{z[L(\alpha, \beta) f(z)]^{\prime}}{L(\alpha, \beta) f(z)}=\Phi(w(z))
$$

Now as

$$
\left[\frac{2 p(z)}{1+p(z)}\right]^{\frac{1}{2}}=\left[2-\frac{2}{1+p(z)}\right]^{\frac{1}{2}}
$$

so, we have

$$
\begin{aligned}
{\left[\frac{2 p(z)}{1+p(z)}\right]^{\frac{1}{2}}=} & 1+\frac{1}{4} p_{1} z+\left(\frac{1}{4} p_{2}-\frac{5}{32} p_{1}^{2}\right) z^{2} \\
& +\left(\frac{1}{4} p_{3}-\frac{5}{16} p_{1} p_{2}+\frac{13}{128} p_{1}^{3}\right) z^{3}+\cdots
\end{aligned}
$$

Similarly,

$$
\begin{aligned}
\frac{z[L(\alpha, \beta) f(z)]^{\prime}}{L(\alpha, \beta) f(z)}= & 1+\frac{\alpha}{\beta} a_{2} z+\left[\frac{\alpha(\alpha+1)}{\beta(\beta+1)} 2 a_{3}-\frac{\alpha^{2}}{\beta^{2}} a_{2}^{2}\right] z^{2} \\
& +\left[3 a_{4}\left(\frac{\alpha(\alpha+1)(\alpha+2)}{\beta(\beta+1)(\beta+2)}\right)-3 a_{2} a_{3} \frac{\alpha^{2}(\alpha+1)}{\beta^{2}(\beta+1)}+a_{2}^{3} \frac{\alpha^{3}}{\beta^{3}}\right] z^{3}+\cdots
\end{aligned}
$$

Thus,

$$
\begin{gathered}
a_{2}=\frac{1}{4} \frac{\beta}{\alpha} p_{1} \\
a_{3}=\frac{\beta(\beta+1)}{\alpha(\alpha+1)}\left[\frac{1}{8} p_{2}-\frac{3}{64} p_{1}^{2}\right]
\end{gathered}
$$

and

$$
a_{4}=\frac{\beta(\beta+1)(\beta+2)}{\alpha(\alpha+1)(\alpha+2)}\left[\frac{1}{12} p_{3}-\frac{7}{96} p_{1} p_{2}+\frac{13}{768} p_{1}^{3}\right]
$$


Now, making use of Equations (6) and (7), we have

$$
\left|a_{3}-\mu a_{2}^{2}\right|=\frac{1}{8} \frac{\beta(\beta+1)}{\alpha(\alpha+1)}\left|p_{2}-\frac{1}{8}\left[4 \mu \frac{\beta(\alpha+1)}{\alpha(\beta+1)}+3\right] p_{1}^{2}\right| .
$$

Using Lemma 1 in conjunction with Equation (9), we obtained the require result.

Theorem 2. Let, for any complex number $\mu, L(\alpha, \beta) f(z) \in S L^{*}(\alpha, \beta)$ having the form Equation (5). Then

$$
\left|\frac{\alpha(\alpha+1)}{\beta(\beta+1)} a_{3}-\mu a^{2} \frac{\alpha^{2}}{\beta^{2}}\right| \leq \frac{1}{4} \max \left(1 ;\left|\mu \frac{\beta(\beta+1)}{\alpha(\alpha+1)}-\frac{1}{4}\right|\right) .
$$

Proof. The proof of this theorem is simple, so we omit the proof.

\section{Special Cases:}

1. For $L(\alpha, \alpha)$ we get;

$$
\left|a_{3}-\mu a_{2}^{2}\right| \leq \frac{1}{4} \max \left(1 ;\left|\mu-\frac{1}{4}\right|\right),
$$

which is proved by Raza and Malik [15].

2. For $L(\alpha, \alpha)$ and $\mu=1$, we can get $H_{2}(1)$.

Theorem 3. Assume that $L(\alpha, \beta) f \in S L^{*}(\alpha, \beta)$ is in the form Equation (5). Then

$$
\left|a_{2} a_{4}-a_{3}^{2}\right| \leq \frac{1}{16}\left(\frac{\beta(\beta+1)}{\alpha(\alpha+1)}\right)^{2} \text {. }
$$

Proof. By make use of Equations (6)-(8), we have

$$
\begin{aligned}
a_{2} a_{4}-a_{3}^{2} & =\left(\frac{\beta}{4 \alpha} p_{1}\right)\left(\frac{\beta(\beta+1)(\beta+2)}{\alpha(\alpha+1)(\alpha+2)}\right)\left(\frac{1}{12} p_{3}-\frac{7}{96} p_{1} p_{2}+\frac{13}{768} p_{1}^{3}\right) \\
& -\left[\frac{\beta(\beta+1)}{\alpha(\alpha+1)}\left(\frac{1}{8} p_{2}-\frac{3}{64} p_{1}^{2}\right)\right]^{2} .
\end{aligned}
$$

After simplification, we have

$$
\begin{aligned}
a_{2} a_{4}-a_{3}^{2} & =\frac{\beta^{2}(\beta+1)}{12288 \alpha^{2}(\alpha+1)}\left[\frac{256(\beta+2)}{(\alpha+2)} p_{1} p_{3}-\frac{192(\beta+1)}{(\alpha+1)} p_{2}^{2}\right. \\
& \left.+\left(\frac{224(\beta+2)}{(\alpha+2)}+\frac{144(\beta+1)}{(\alpha+1)}\right) p_{1}^{2} p_{2}+\left(\frac{52(\beta+2)}{(\alpha+2)}-\frac{27(\beta+1)}{(\alpha+1)}\right) p_{1}^{4}\right] .
\end{aligned}
$$

By substituting values of $p_{2}$ and $p_{3}$ from Lemma 3, after some simplification, we have

$$
\begin{aligned}
a_{2} a_{4}-a_{3}^{2} & \leq \frac{\beta^{2}(\beta+1)}{12288 \alpha^{2}(\alpha+1)}\left[\left(\frac{4(\beta+2)}{(\alpha+2)}-\frac{3(\beta+1)}{(\alpha+1)}\right) p_{1}^{4}\right. \\
& +\frac{128(\beta+2)}{(\alpha+2)}\left(4-p_{1}^{2}\right)+\left(\frac{24(\beta+1)}{(\alpha+1)}-\frac{16(\beta+2)}{(\alpha+2)}\right) \\
& \left(4-p_{1}^{2}\right) p_{1}^{2} \rho+\rho^{2}\left(4-p_{1}^{2}\right) \\
& \left.\left\{\left(\frac{64(\beta+2)}{(\alpha+2)}-\frac{48(\beta+1)}{(\alpha+1)}\right) p_{1}^{2}+\frac{192(\beta+1)}{(\alpha+1)}\right\}\right],
\end{aligned}
$$

or by considering right-hand side as $F\left(p_{1}, \rho\right)$, we can write

$$
a_{2} a_{4}-a_{3}^{2}=F\left(p_{1}, \rho\right) \text {. }
$$


Differentiating w.r.t. $\rho$, assuming $\rho>0$ and taking $p_{1}=p \in[0,2]$, we can obtain

$$
\begin{aligned}
\frac{\partial F(p, \rho)}{\partial p} & =\frac{\beta^{2}(\beta+1)}{12288 \alpha^{2}(\alpha+1)}\left[\left(\frac{24(\beta+1)}{(\alpha+1)}-\frac{16(\beta+2)}{(\alpha+2)}\right)\right. \\
& \left(4-p^{2}\right) p^{2}+2 \rho\left(4-p^{2}\right) \\
& \left.\left\{\left(\frac{64(\beta+2)}{(\alpha+2)}-\frac{48(\beta+1)}{(\alpha+1)}\right) p^{2}+\frac{192(\beta+1)}{(\alpha+1)}\right\}\right] .
\end{aligned}
$$

As $\frac{\partial F(p, \rho)}{\partial p}>0$, we then find that $F(p, \rho)$ increases on $[0,1]$. Hence,

$$
F(p))=F(p, 1)=\max F(p, \rho) .
$$

For $p=0$, we can write

$$
\left|a_{2} a_{4}-a_{3}^{2}\right| \leq \frac{1}{16}\left(\frac{\beta(\beta+1)}{\alpha(\alpha+1)}\right)^{2},
$$

which is the desired result.

\section{Special Case:}

If we put $\alpha=\beta$, then for $L(\alpha, \alpha)$, we can obtain

$$
\left|a_{2} a_{4}-a_{3}^{2}\right|=\frac{1}{16}
$$

which is proved by Raza and Malik [15].

Theorem 4. Let $L(\alpha, \beta) f \in S L^{*}(\alpha, \beta)$ is in the form Equation (5). Then

$$
\left|a_{2} a_{4}-a_{4}\right| \leq \frac{1}{6}\left(\frac{\beta(\beta+1)(\beta+2)}{\alpha(\alpha+1)(\alpha+2)}\right)^{2} .
$$

Proof. Using Lemma 3, we can write

$$
\begin{aligned}
a_{2} a_{4}-a_{4}= & \frac{\beta(\beta+1)}{\alpha(\alpha+1)}\left[\left(\frac{\beta}{32 \alpha}-\frac{7(\beta+2)}{96(\alpha+2)}\right) p_{1} p_{2}\right. \\
& \left.+\left(-\frac{3 \beta}{256 \alpha}+\frac{13(\beta+2)}{768(\alpha+2)}\right) p_{1}^{3}-\frac{\beta+2}{12(\alpha+2)} p_{3}\right] .
\end{aligned}
$$

By putting values of $p_{2}$ and $p_{3}$, we can obtain

$$
\begin{aligned}
a_{2} a_{4}-a_{4}= & \frac{\beta(\beta+1)}{\alpha(\alpha+1)}\left[\left(\frac{9 \beta}{\alpha}-\frac{31(\beta+2)}{(\alpha+2)}\right) p 1^{3}\right. \\
& +\left(\frac{9 \beta}{\alpha}-\frac{60(\beta+2)}{(\alpha+2)}\right)\left(4-p_{1}^{2}\right) x p_{1} \\
& \left.+\frac{16(\beta+2)}{(\alpha+2)}\left(4-p_{1}^{2}\right) p_{1} x^{2}-\frac{32(\beta+2)}{(\alpha+2)}\left(4-p_{1}^{2}\right)\left(1-|x|^{2}\right) z\right] .
\end{aligned}
$$

Now, using triangular inequality, replacing $|x|$ with $\rho$, assuming $p_{1}=p$ and differentiating w.r.t $\rho$ after simplification, we obtain

$$
\begin{gathered}
F_{1}(p)=G_{1}(p)=\frac{\beta(\beta+1)}{768 \alpha(\alpha+1)}\left[\left(\frac{9 \beta}{\alpha}-\frac{31(\beta+2)}{(\alpha+2)}\right) p^{3}+\frac{32(\beta+2)}{(\alpha+2)}\left(4-p_{2}\right)\right], \\
G_{1}^{\prime}(p)=\frac{\beta(\beta+1)}{768 \alpha(\alpha+1)}\left[\left(3 \frac{9 \beta}{\alpha}-\frac{31(\beta+2)}{(\alpha+2)}\right) p^{2}-\left(\frac{64(\beta+2)}{(\alpha+2)}\right) p\right]
\end{gathered}
$$


and

$$
G_{1}^{\prime \prime}(p)=\frac{\beta(\beta+1)}{768 \alpha(\alpha+1)}\left[\left(6 \frac{9 \beta}{\alpha}-\frac{31(\beta+2)}{(\alpha+2)}\right) p-\frac{64(\beta+2)}{(\alpha+2)}\right]<0 .
$$

For $p=0$, we can get

$$
G_{1}(0)=\frac{128 \beta(\beta+1)(\beta+2)}{768 \alpha(\alpha+1)(\alpha+2)}
$$

or

$$
G_{1}(0)=\frac{\beta(\beta+1)(\beta+2)}{6 \alpha(\alpha+1)(\alpha+2)}
$$

Theorem 5. Let $L(\alpha, \beta) f \in S L^{*}(\alpha, \beta)$ be the form Equation (5). Then

$$
\left|H_{3}(1)\right| \leq \frac{1}{576}\left[\frac{9 \beta^{2}(\beta+1)^{2}}{\alpha^{2}(\alpha+1)^{2}}+\frac{16 \beta(\beta+1)(\beta+2)}{\alpha(\alpha+1)(\alpha+2)}+18\right] .
$$

Proof. As,

$$
a_{3}\left(a_{2} a_{4}-a_{3}^{2}\right)-a_{4}\left(a_{4}-a_{2} a_{3}\right)+a_{5}\left(a_{1} a_{3}-a_{2}^{2}\right)=H_{3}(1) .
$$

By applying triangular inequality; it gives

$$
\left|a_{2} a_{4}-a_{3}^{2}\right|\left|a_{3}\right|+\left|a_{2} a_{3}-a_{4}\right|\left|a_{4}\right|+\left|a_{1} a_{3}-a_{2}^{2}\right|\left|a_{5}\right|=\left|H_{3}(1)\right| .
$$

After simplification, we can write

$$
\left|H_{3}(1)\right| \leq \frac{1}{4}\left(\frac{\beta^{2}(\beta+1)^{2}}{16 \alpha^{2}(\alpha+1)^{2}}\right)+\frac{1}{6}\left(\frac{\beta(\beta+1)(\beta+2)}{6 \alpha(\alpha+1)(\alpha+2)}\right)+\left(\frac{1}{8} \frac{1}{4}\right) .
$$

Hence,

$$
\left|H_{3}(1)\right| \leq \frac{1}{576}\left[\frac{9 \beta^{2}(\beta+1)^{2}}{\alpha^{2}(\alpha+1)^{2}}+\frac{16 \beta(\beta+1)(\beta+2)}{\alpha(\alpha+1)(\alpha+2)}+18\right] .
$$

\section{Conclusions}

In this work, we introduced a new subclass of univalent function associated with a Carlson-Shaffer operator, named as $S L^{*}(\alpha, \beta)$. By applying the Carlson-Shaffer operator, we derived an upper bound of $H_{3}(1)$ of the desired subclass associated to the right half of the lemniscate of Bernoulli. Certain properties such as: upper bound of $H_{3}(1)$, coefficient estimate, etc. for this newly defined subclass have also been discussed in detail. We also compare the obtained results with known results in special cases.

Author Contributions: Conceptualization, I.A.; Formal analysis, N.U. and I.A.; funding acquisition, J.-S.R.; Investigation, N.U.; Methodology, N.U., I.A. and B.K.; Supervision, I.A. and S.M.H.; visualization, S.M.H.; N.K. and J.-S.R.; writing—original draft, N.U.; I.A. and B.K.; writing-review \& editing, S.M.H.; N.K. and J.-S.R. All authors have read and agreed to the published version of the manuscript.

Funding: This research was supported by: 1. The Basic Science Research Program, through the National Research Foundation of Korea, funded by the Ministry of Education (2016R1D1A1B01008058). 2. The Competency Development Program for Industry Specialists of the Korean Ministry of Trade, Industry and Energy (MOTIE), operated by the Korea Institute for Advancement of Technology (KIAT) (No. P0002397, HRD program for Industrial Convergence of Wearable Smart Devices).

Institutional Review Board Statement: Not applicable.

Informed Consent Statement: Not applicable.

Acknowledgments: The authors would like to acknowledge the Balochistan University of Information Technology, Engineering and Management Sciences (BUITEMS) for providing research facilities and an excellent environment. 
Conflicts of Interest: The authors declare no conflict of interest.

\section{References}

1. Sokol, J. Coefficient estimates in a class of strongly starlike functions. Kyungpook Math. J. 2009, 49, 349-353. [CrossRef]

2. Sokół, J.; Stankiewicz, J. Radius of convexity of some subclasses of strongly starlike functions. Zesz. Nauk. Politech. Rzesz. Mat 1996, 19, 101-105.

3. Güney, H.Ö.; Murugusundaramoorthy, G.; Srivastava, H.M. The second hankel determinant for a certain class of bi-close-toconvex functions. Results Math. 2019, 74, 93. [CrossRef]

4. Janteng, A.; Halim, S.A.; Darus, M. Coefficient inequality for a function whose derivative has a positive real part. J. Inequal. Pure Appl. Math. 2006, 7, 1-5.

5. Mishra, A.K.; Gochhayat, P. Second hankel determinant for a class of analytic functions defined by fractional derivative. Int. J. Math. Math. Sci. 2008, 2008, 153280. [CrossRef]

6. Singh, G.; Singh, G. On the second hankel determinant for a new subclass of analytic functions. J. Math. Sci. Appl. 2014, 2, 1-3.

7. Srivastava, H.M.; Ahmad, Q.Z.; Khan, N.; Khan, N.; Khan, B. Hankel and toeplitz determinants for a subclass of q-starlike functions associated with a general conic domain. Mathematics 2019, 7, 181. [CrossRef]

8. Srivastava, H.; Raza, N.; AbuJarad, E.S.; Srivastava, G.; AbuJarad, M.H. Fekete-szegö inequality for classes of (p, q)-starlike and (p, q)-convex functions. Rev. Real Acad. Cienc. Exactas Físicas Nat. Ser. A Matemáticas 2019, 113, 3563-3584. [CrossRef]

9. Babalola, K.O. On $h \_3(1)$ hankel determinant for some classes of univalent functions. arXiv 2009, arXiv:0910.3779.

10. Mahmood, S.; Srivastava, H.M.; Khan, N.; Ahmad, Q.Z.; Khan, B.; Ali, I. Upper bound of the third hankel determinant for a subclass of q-starlike functions. Symmetry 2019, 11, 347. [CrossRef]

11. Srivastava, H.M.; Ahmad, Q.Z.; Darus, M.; Khan, N.; Khan, B.; Zaman, N.; Shah, H.H. Upper bound of the third hankel determinant for a subclass of close-to-convex functions associated with the lemniscate of bernoulli. Mathematics 2019, 7, 848. [CrossRef]

12. Shi, L.; Srivastava, H.M.; Arif, M.; Hussain, S.; Khan, H. An investigation of the third hankel determinant problem for certain subfamilies of univalent functions involving the exponential function. Symmetry 2019, 11, 598. [CrossRef]

13. Ma, W. A unified treatment of some special classes of univalent functions. In Proceedings of the Conference on Complex Analysis; International Press Inc.: Somerville, MA, USA, 1992.

14. Grenander, U.; Szegö, G. Toeplitz Forms and Their Applications; University of California Press: Berkeley, CA, USA, 1958.

15. Raza, M.; Malik, S.N. Upper bound of the third hankel determinant for a class of analytic functions related with lemniscate of bernoulli. J. Inequalities Appl. 2013, 2013, 412. [CrossRef] 